\title{
LA DESCRIPCIÓN ARCHIVÍSTICA. DE LOS INSTRUMENTOS DE DESCRIPCIÓN HACIA LA WEB SEMÁNTICA
}

\author{
Julián Moyano Collado* \\ Especialista en archivos y gestión documental
}

\begin{abstract}
Resumen: El texto realiza una aproximación a la descripción como proceso de trabajo en los archivos, recogiendo los frutos de esta actividad que son los instrumentos de descripción, y las posibilidades que brinda la tecnología web, mediante los Sistemas de Acceso Archivístico y con especial mención a la Web Semántica. Para acercarnos al amplio concepto de la descripción, entendida como proceso, resultado e instrumento, se recogen algunos apuntes teóricos fundamentados por diversos autores y ejemplos que permitan entender la importancia de esta tarea en el actual entorno electrónico.

Palabras clave: Descripción archivística; instrumentos de descripción; Sistemas de Acceso Archivístico; Sistemas de Información Archivística; normalización descriptiva; ISAD (G); ISAAR (CPF); EAC; EAD; web; Web Semántica.
\end{abstract}

Title: ARCHIVAL DESCRIPTION: FROM ARCHIVAL FINDING AIDS TO SEMANTIC WEB.

Abstract: The paper is an approach for description as work in the Archives, documenting the results of this activity (archival finding aids) and the possibilities offered by web technology (Archives Access Systems) with special reference to the semantic web. Also, this paper shows some theoretical issues substantiated by authors and examples to understand the broad concept of description, as a process, outcome and finding aid, and to understand the importance of this task in an electronic environment.

Keywords: Archival description; archival finding aids; Archives Access Systems; Information Archival Systems; standards for archival description; ISAD (G); ISAAR (CPF); EAC; EAD; Web; Semantic Web.

\section{INTRODUCCIÓN.}

El documento de archivo se relaciona con información descriptiva que hace posible su identificación, localización, recuperación o disponibilidad. Esa información, además de acompañar al documento, le aporta nuevos datos que sirven para representar su contenido, determinar su utilidad y hacer posible su correcta gestión.

Los archivos disponen de elementos y herramientas que ofrecen información descriptiva de su documentación, siendo, en el actual entorno electrónico donde la descripción se hace todavía mucho más importante puesto que va a permitir el uso de los documentos en redes e infraestructuras universales de comunicación. Pero la utilización de las descripciones no resulta sencilla ante la cantidad de sistemas, normas y lenguajes de comunicación surgidos en los últimos años para facilitar el intercambio de información descriptiva de los archivos. Este gran número de posibilidades puede ser un inconveniente para la difusión de los recursos de los archivos y por esa razón estas líneas pretenden dar a conocer la importancia de la descripción como un proceso y un resultado del trabajo en los archivos utilizando los medios que permiten las tecnologías y la Web Semántica. Para ello se analizan fuentes, estudios y normas relacionadas con esta materia, y se dibuja una evolución desde el proceso descriptivo tradicional hasta los actuales servicios electrónicos de la web, buscando el enfoque de una red más útil y funcional, como es la Web Semántica.

El análisis planteado muestra la relación entre el proceso descriptivo en los archivos y la Web Semántica, tomando en consideración dos puntos de vista, uno teórico y otro práctico:

- Teórico. La teoría es necesaria para fundamentar la descripción como uno de los pilares de la Archivística. Para ello se recogen trabajos de diversos autores en este campo que permitan justificar la actividad descriptiva como una actividad elemental para el tratamiento de la documentación. Este punto de vista teórico explica el concepto de descripción y su proceso, mostrando los recursos producidos como los instrumentos de descripción y los Sistemas de Acceso Archivísticos.

- Práctico. La parte práctica intenta ofrecer mediante ejemplos y exposiciones cómo funciona el proceso descriptivo en los archivos y cuál es su resultado. Estos ejemplos pretenden ser lo más simples y representativos posibles para dar a conocer los distintos resultados y funciones.

\footnotetext{
*julianmoyanoc@gmail.com
} 
Estos dos puntos de vista permiten aunar los resultados del proceso descriptivo respaldado por los estándares propios de los archivos y las tecnologías, mostrando así una evolución de las posibilidades de esta tarea para justificar que el entorno semántico puede ser una gran ayuda para difundir y ofrecer los recursos archivísticos de una manera más global.

\section{2. ¿QUÉ ES DESCRIBIR?: UN ACERCAMIENTO A LA DESCRIPCIÓN Y SU OBJETO.}

Para comprender el proceso descriptivo hay que empezar por plantear la definición de un término tan genérico y habitual como es describir, acción que seguramente se pone en práctica en innumerables momentos de la vida cotidiana. Entender y conocer su significado en el entorno de los archivos obliga a recurrir a algunas de las referencias más actuales relacionadas con este ámbito, como por ejemplo el Manual de descripción multinivel que define describir como la acción consistente en representar estructuradamente los materiales archivísticos ${ }^{\mathrm{i}}$.

También la web de la Society of American Archivist (SAA) ofrece otra definición interesante de la descripción, entendida como la creación de una representación precisa del material archivístico mediante procesos de captura, recopilación, análisis y organización de la información que servirá para identificar el material archivistico y explicar el contexto y sistema que lo creó $^{2}$. SAA afirma que describir es crear una representación precisa (accurate) y exacta de los documentos para identificarlos e interpretar su realidad.

En los archivos, el proceso descriptivo tiene una clara connotación práctica y resulta fundamental para gestionar adecuadamente los documentos al poder ofrecer con el resultado de esta tarea un correcto servicio.

El trabajo de José Luis Bonal $\mathrm{Zazo}^{3}$ analiza a través de diversos autores y expertos el resultado del proceso descriptivo, diferenciando dos posibilidades:

- $\quad$ El proceso de descripción que produce instrumentos de descripción

- La descripción que se utiliza como representación de los documentos

Para llegar a estas conclusiones se ha basado en especialistas como Theodore Schellenberg y Antonia Heredia que identifican describir como el conjunto de actividades destinadas al acceso a la documentación, y ese acceso se logra gracias al desarrollo de los instrumentos de descripción correspondientes que facilitarán la información necesaria sobre los documentos.

Pedro López y Olga Gallego, también citan la obra de Bonal Zazo, identifican la descripción de las unidades documentales desde otra perspectiva, para ellos es crear una enumeración de sus atributos, es decir, la función de representación de los documentos.

Esta aproximación teórica a la descripción archivística se centra en las unidades documentales o materiales archivísticos como objetos del proceso descriptivo, y casi deducimos que se refiere a los documentos, series, secciones, fondos u otras unidades que componen los archivos.

Y además otros autores, como Javier Barbadillo, añaden a esa visión nuevos detalles al considerar la descripción archivística como el registro de información estructurada sobre los documentos de archivo y su contexto que sirve para cumplir los fines del sistema de archivos ${ }^{4}$. Barbadillo menciona registro de información, entendido como producción o creación de nuevos datos estructurados sobre los documentos o su contexto. Y el contexto indica que es un objeto más de la descripción que puede ofrecer información sobre el cómo se ha producido el documento, quién lo ha producido y el por qué de su existencia, preguntas que no suele responder el propio contenido de las agrupaciones documentales.

En esta misma línea Ramón Martín Suquía define la descripción, como el conjunto de actividades destinadas a la representación intelectual del conjunto de unidades de información manejadas en los archivos, así como el resultado concreto fruto de esas actividades ${ }^{5}$. Martín Suquía habla de unidades de información, expresión más acorde con la actualidad y lo suficientemente amplia para que integre en el proceso descriptivo los múltiples elementos de información que componen los archivos.

Un archivo no sólo puede ofrecer descripciones de sus agrupaciones o unidades documentales, también debe aclarar qué organizaciones son sus productoras, o los procedimientos que los generaron, para así tener la máxima información sobre los componentes relacionados con el organismo, que permitirán su correcto funcionamiento y gestión. 
Estas aproximaciones al término descripción pertenecen a reconocidos profesionales o asociaciones expertas en la materia. Por otra parte, también se ha logrado consensuar el término para establecer su significado mediante la inclusión de la definición en el apartado destinado al glosario de diferentes normas internacionales de archivos como ISAD (G), ISAAR (CPF) o ISDF ${ }^{6}$. Las normas han tenido una amplia aceptación para regular la manera de crear y ofrecer las descripciones de agrupaciones documentales, entidades y funciones respectivamente que están disponibles en los centros archivísticos.

Estas normas recogen en su glosario descripción archivística, definida como la elaboración de una representación exacta de la unidad de descripción y, en su caso, de las partes que la componen mediante la recopilación, análisis, organización y registro de la información que sirve para identificar, gestionar, localizar y explicar los documentos de archivo, así como su contexto y el sistema que los ha producido. El término sirve también para describir los resultados de este proceso. (Archival description) ${ }^{7}$.

Un paso más en la normalización de las descripciones archivísticas es la planteada recientemente por la Comisión de Normas Españolas de Descripción Archivística CNEDA $^{8}$ en el Modelo Conceptual de Descripción Archivística y Requisitos de Datos Básicos de las Descripciones de Documentos de Archivo, Agentes y Funciones. En este documento se define descripción archivística como la elaboración de representaciones de documentos de archivo y, en su caso, de otras entidades archivísticas (agentes, funciones, etc.) $)^{9}$ y también recoge las diferentes unidades de información archivísticas que tienen la posibilidad de ser descritas como son los agentes (instituciones, personas o familias) o las funciones, además de las agrupaciones documentales, denominando entidad al elemento descriptible e identificable de un archivo mediante la asignación de atributos y relaciones.

Estas referencias demuestran que las descripciones de agrupaciones documentales han sido desde siempre las más producidas y utilizadas en cualquier archivo, siendo el producto por excelencia de estos centros, pero en los últimos años se han empezado a normalizar y desarrollar las descripciones de agentes y funciones. Éstas últimas ofrecen información que ayudan a precisar todavía más las descripciones de las unidades documentales y sirven para relacionar los documentos entre ellos, atendiendo a características de su entorno como los agentes productores o las funciones que generaron el documento.

A pesar de estas definiciones y teorías hay que entender que describir es mucho más que lo expresado en cualquiera de las afirmaciones expuestas, es quizá el trabajo más importante que se realiza en un archivo. De su acertado desarrollo dependerá la posterior recuperación y uso de los documentos. Una descripción manual o automatizada que no sea correcta puede suponer la pérdida irreversible del documento o de la información, y una adecuada creación de descripciones normalizadas mejorarán el intercambio de datos e información, fundamental en el actual mundo tecnológico y de interoperabilidad en el que nos encontramos. En definitiva, el proceso descriptivo va a permitir ofrecer por parte de los archivos servicios de calidad acordes con las actuales demandas de la sociedad y de los ciudadanos del siglo XXI, pero debe seguir una serie de pasos y procedimientos que se presentarán a continuación.

\section{EL PROCESO DESCRIPTIVO.}

Las unidades o agrupaciones documentales se generan por la reunión de los documentos provenientes de un organismo o fruto de una función administrativa, siendo el principio de procedencia el marco de trabajo.

La descripción archivística, según ISAD $(G)$ y otras normas internacionales, se obtiene al realizar un trabajo previo que permita representar intelectualmente y con precisión una agrupación, entidad o función del archivo. Ese trabajo consiste en obtener información de los elementos a describir, mediante:

- La recopilación. Que consiste en reunir diferentes datos e información sobre los elementos que se desean representar o describir. Los datos pueden ser textuales, numéricos, o cronológico, expresados en lenguaje natural o a través de códigos preestablecidos e incluso pueden incluir una reproducción o copia del documento a describir. Recopilar obliga a capturar todos los datos e información posible, pero no de una manera arbitraria, se debe seguir un procedimiento determinado por las normas y manuales de referencia.

- El análisis. Estudia las entidades a través de los datos recopilados, para determinar los más relevantes que ofrecen el contenido y una representación intelectual adecuada de las mismas. El análisis va a seleccionar la información que ofrece una caracterización útil y objetiva sobre la unidad o el elemento descrito.

- La organización. Es el procedimiento físico e intelectual, así como su resultado, consistente en analizar y disponer las unidades de descripción para su posterior utilización ${ }^{10}$.

- El registro. No se debe confundir esta acción, con ningún acto administrativo. La tarea se refiera a que la información recogida, analizada y organizada obtenida de las entidades esté disponible para su posterior uso. 
Para ello es necesario que sea anotada y guardada de forma apropiada. Si durante años se han utilizado tarjetas catalográficas o libros, hoy en día las bases de datos son la herramienta para disponer con posterioridad de toda la información obtenida del proceso descriptivo.

Todo este conjunto de tareas permitirán obtener la información suficiente de los documentos de archivo y de su contexto para lograr como resultados más destacados una adecuada identificación, gestión y localización, que consisten en:

- Identificación. Entendido como un proceso que servirá para reconocer y diferenciar unas entidades archivísticas con respecto a otras, con la asignación de atributos a las mismas. La identificación debe ser clara, concisa y lo suficientemente extensa para poder distinguir las unidades inequívocamente.

- Gestión. La información obtenida en el proceso descriptivo podrá usarse para la recuperación, conservación, acceso, seguridad, organización de las unidades documentales. Estas actividades, y otras muchas, hacen posible la correcta disponibilidad de los documentos y los materiales de archivo.

- Localización. Con la localización se permitirá ubicar las unidades documentales en un espacio físico o electrónico a través de la asignación de una signatura o código que permita su posterior recuperación.

El conjunto de información obtenido ofrece y representa las agrupaciones documentales $u$ otras entidades, dibujando la naturaleza y génesis de los documentos. Si las descripciones de las agrupaciones documentales muestran una realidad sobre su contenido y características, las descripciones de agentes y funciones ofrecen el contexto que ha generado esas agrupaciones. El contexto es el conjunto de circunstancias que rodean la creación del documento ${ }^{11}$ y sirve para entender 'quién' produce los documentos (agentes) y 'cómo' se han generado (funciones).

Las agrupaciones documentales están integradas generalmente por fondos, secciones, subsecciones, series, unidades de instalación, unidades documentales simples y unidades documentales compuestas que independientemente de su clasificación y proceso, necesitan someterse a un proceso descriptivo que permita su identificación, recuperación y uso.

Para describir las agrupaciones documentales es necesario utilizar la norma marco internacional creada para este fin: ISAD (G), o en su caso y si es posible la norma nacional o autonómica que ha perfilado mejor ese estándar. ISAD (G) define la estructura y los componentes descriptivos necesarios de cualquier agrupación documental. Esta norma se compone de siete áreas que agrupan 26 elementos, atendiendo al contenido que representan y la relación entre ellos. Uno de sus puntos más destacables es la relación que se genera entre los diferentes elementos descritos, conformando una estructura multinivel ${ }^{12}$, que recorre desde lo general (fondo) a lo específico (unidad documental simple). La representación multinivel ofrece información pertinente en cada nivel de descripción (fondo, sección, unidad documental...) y permite conectar o vincular las descripciones sin repetir la información.

La descripción archivística sigue el denominado efecto Jano ${ }^{13}$, muy conocido en el desarrollo de infraestructuras de datos espaciales. El efecto Jano consiste en ofrecer un determinado nivel jerárquico, por ejemplo una sección, en relación con sus dos visiones distintas posibles. Una de esas visiones, proporciona una perspectiva global desde un nivel más alto de la descripción, que en el caso de tratarse de una sección, sería el fondo; y la otra visión se enfoca hacia las partes de sus niveles más bajos, que serían subsecciones y series respectivamente.

También pueden existir dos puntos de vista de esta jerarquía o representación multinivel. El primero de ellos tiene que ver con su nivel superior, por ejemplo el nivel fondo, abarca todos los componentes de niveles inferiores (secciones, subsecciones, series...). El segundo punto de vista hace referencia a que cualquier nivel de un archivo, por ejemplo una serie, sirve como punto de partida para otros niveles superiores, por ejemplo la sección o subsección a la que pertenece. La descripción multinivel desarrollada, crea un entorno en el cual la información de cada agrupación documental recurre a los datos de otras agrupaciones; en función de las necesidades del usuario o el uso del sistema de información ${ }^{14}$.

La norma ISAD (G), y todos sus desarrollos nacionales y autonómicos, son el resultado de años de trabajo de numerosos profesionales de los archivos con la documentación y con los problemas derivados de la falta de estándares que impedían un correcto uso de la información descriptiva obtenida de los documentos. Sin querer profundizar en la norma ISAD $(\mathrm{G})$ se muestran a continuación algunos ejemplos fruto de un proceso descriptivo de varias agrupaciones documentales, pero será en el apartado 4 Los Sistemas de Acceso, la Web y las tecnologías semánticas donde se profundiza más en la utilidad y problemas de este tipo de estructuras, al compararse con otras formas de ofrecer la información. El ejemplo de a continuación presenta el resultado de obtener información después de recopilar, analizar, organizar y registrar las agrupaciones documentales, obteniendo el siguiente resultado estructurado y normalizado en una sencilla tabla que permitará comprender el contenido de las unidades representadas ${ }^{15}$. 


\begin{tabular}{|l|l|}
\hline \multicolumn{1}{|c|}{ Elementos } & \multicolumn{1}{c|}{ Información descriptiva } \\
\hline 1.1. Código de referencia & ES/AHPZ - A/002446 \\
\hline 1.2. Título & Delegación Provincial de Hacienda de Zaragoza \\
\hline 1.3. Fecha(s) & $1881-1992$ \\
\hline 1.4. Nivel de descripción & Fondo \\
\hline 1.5 Volumen y soporte de la unidad de descripción & 1110 cajas. 1473 libros \\
\hline 2.1 Nombre del o de los productor(es) & Delegación Provincial de Hacienda de Zaragoza \\
\hline
\end{tabular}

Tabla I. Descripción de una agrupación documental: Fondo.

\begin{tabular}{|l|l|}
\hline \multicolumn{1}{|c|}{ Elementos } & \multicolumn{1}{c|}{ Información descriptiva } \\
\hline 1.1. Código de referencia & ES/AHPZ - A/002446/000004 \\
\hline 1.2. Título & $\begin{array}{l}\text { Expediente del Administrador de las Rentas Estancadas de } \\
\text { Tarazona, Antonio Morales. }\end{array}$ \\
\hline 1.3. Fecha(s) & 1886 \\
\hline 1.4. Nivel de descripción & Unidad documental compuesta \\
\hline 1.5 Volumen y soporte de la unidad de descripción & 1 exp. \\
\hline 2.1 Nombre del o de los productor(es) & Administración Provincial de Hacienda de Zaragoza \\
\hline
\end{tabular}

Tabla II. Descripción de una agrupación documental: Unidad documental compuesta.

La utilidad y servicio de esta descripción dependerá del análisis previo, dedicación y medios empleados con la documentación por los archiveros y profesionales para generar la información mostrada. Si la información es precisa, clara y exhaustiva facilitará su tratamiento y uso por parte de usuarios, otros profesionales y también máquinas permitiendo una adecuada identificación, gestión y localización.

Los dos ejemplos mostrados se relacionan entre sí jerárquicamente, al ser la unidad documental compuesta (Expediente del Administrador...) una parte del fondo (Delegación Provincial de Hacienda de Zaragoza), cumpliendo con la estructura multinivel citada. La suma de las columnas, Elementos e Información descriptiva, componen un registro, y cada fila representa un campo. Estos dos conceptos, registro y campo, forman las unidades de cualquier base de datos que las tecnologías de tratamiento de la información utilizan normalmente. Y así durante el proceso descriptivo se ha generado una base de datos, que puede estar reflejada mediante la siguiente tabla:

\begin{tabular}{|c|c|c|c|c|c|c|}
\hline 4 & A & B & c & D & E & $\mathrm{F}$ \\
\hline 1 & 1.1. Código de referencia & 1.2. Título & 1.3. Fecha(s) & 1.4. Nivel de descripción & 1.5 Volumen $y$ & 2.1 Nombre del o de los productor(es) \\
\hline 2 & ES/AHPZ - A/002446 & $\begin{array}{l}\text { Delegación Provincial de Hacienda de } \\
\text { Zaragoza }\end{array}$ & 1881-1992 & Fondo & $\begin{array}{c}1110 \text { cajas. } 1473 \\
\text { libros }\end{array}$ & Delegación Provincial de Hacienda de Zaragoza \\
\hline 3 & $\begin{array}{c}\text { ES/AHPZ- } \\
\text { A/002446/000004 }\end{array}$ & $\begin{array}{c}\text { Expediente del Administrador de las } \\
\text { Rentas Estancadas de Tarazona, } \\
\text { Antonio Morales. }\end{array}$ & 1886 & Unidad documental compuesta & 1 exp. & Administración Provincial de Hacienda de Zaragoza \\
\hline 4 & & & & & & \\
\hline 5 & & & & & & \\
\hline
\end{tabular}

Figura 1. Representación en una tabla de las descripciones.

Las bases de datos componen una colección de datos estructurados según un modelo que refleje las relaciones y restricciones existentes en el mundo real ${ }^{l 6}$. Y con esta definición se puede afirmar que la colección de datos estructurados ${ }^{17}$ representa a la norma ISAD $(\mathrm{G})$, y además tiene relaciones jerárquicas siguiendo el modelo multinivel, y restricciones (una unidad documental simple, no puede ser un fondo, por ejemplo). Y el mundo real no es otro que las entidades que forman el archivo.

Las descripciones recogidas en una base de datos han sido producidas en muchos casos por los archiveros tras un proceso de análisis de la información existente en las agrupaciones documentales y en otros elementos. Fruto de este 
trabajo se generan unas herramientas imprescindibles en un archivo, los instrumentos de descripción que van a presentar los resultados de este proceso.

\section{LOS INSTRUMENTOS DE DESCRIPCIÓN.}

Se ha señalado anteriormente que las bases de datos son la herramienta más representativa en la actualidad del proceso descriptivo, y constituyen el principal punto de acceso a la información contenida en ellos. Pero antes de hablar de bases de datos y sistemas de acceso hay que precisar que cuando se habla de instrumentos de descripción se refiere generalmente a instrumentos de trabajo, definidos como aquellos instrumentos de referencia de los que se sirve el archivero para plasmar en ellos el resultado de su actividad profesional, con la doble finalidad de controlar los fondos a su cargo y dar la máxima difusión al contenido de los mismos, facilitando su conocimiento al investigador o al simple consultante ${ }^{18}$. El proceso descriptivo genera instrumentos de descripción que se pueden diferenciar en dos clases, atendiendo a su utilidad y servicio:

- Servir como instrumento de trabajo y control para el personal del archivo que permita la gestión interna de las agrupaciones documentales, su orden, localización, conservación o información descriptiva relativa a su conservación o también cuestiones de seguridad de los fondos. Estos instrumentos se denominan de control y se componen básicamente de registros, relaciones de entrega y de otros registros con funciones informativas, como por ejemplo conocer el número de usuarios y consultas.

- Publicitar el contenido de las agrupaciones documentales y satisfacer las necesidades de información sobre el archivo de cualquier usuario. Este tipo de instrumento se denomina de información o referencia. Este tipo de instrumentos ofrecen guías, catálogos e inventarios.

A continuación se recopilan brevemente los instrumentos de descripción citados para ofrecer una perspectiva general de los mismos, diferenciarlos con claridad y entender la función de cada uno de ellos:

\section{Instrumentos de control.}

Registro: instrumento de control que ofrece datos básicos relacionados con la fecha de incorporación de una agrupación documental a un archivo. El soporte solía ser un libro de gran formato.

Relación de entrega: documento que ofrece información sobre las agrupaciones documentales transferidas al archivo, así como el número de unidades, tipo e identificación de los responsables del proceso de transferencia, entre otros datos. Un ejemplo de hoja de transferencia puede ser $<$ http://goo.gl/bK8Wq $>$.

Informes y registros de usuarios y consultas: información recogida por cuestiones de seguridad para controlar el acceso a las agrupaciones pero también con finalidades estadísticas sobre los usuarios y las agrupaciones documentales consultadas.

\section{Instrumentos de información.}

Guías: ofrecen información muy general sobre los fondos y agrupaciones documentales de uno o más archivos. También se denomina guías a la recopilación de fuentes documentales especializadas en un periodo, tema o región. Las guías suelen ir acompañadas de información práctica del centro y de los servicios ofrecidos, el personal, sede electrónica o localización. Algunas de las guías atendiendo a sus características:

- Censo guía: información sobre los archivos de un área geográfica determinada. Por ejemplo el Censo Guía de Archivos del Ministerio de Cultura $<$ http://censoarchivos.mcu.es $>$.

- Guía de fuentes: información sobre fuentes documentales y archivos que tratan sobre un tema determinado. Por ejemplo la Guía de fuentes documentales de archivos del Ministerio de Cultura <http://goo.gl/QdE32>.

- Guía de Archivo o Guía del Investigador: mucho más específica y completa que el resto de guías al centrarse en un único centro. La información sin pretender ser exhaustiva ofrece al usuario una visión general del archivo y de sus agrupaciones documentales disponibles. Por ejemplo la Guía del investigador del Archivo Histórico Provincial de Huesca $<$ http://goo.gl/jZjDa $>$.

Catálogos: es un instrumento excepcional y se produce para unidades documentales o agrupaciones muy limitadas con características especiales como pueden ser un conjunto de pergaminos, un número limitado de planos, o fotografías de reconocidos autores, por citar algunos ejemplos. Se describe unidad documental a unidad documental. El catálogo es el instrumento de trabajo característico de una biblioteca, pero no de un archivo. Es una rareza, y como ya se ha indicado sólo se utiliza para casos muy concretos por cuestiones prácticas y de recursos. Un ejemplo de recurso catalogado podría ser: $<\mathrm{http} / / \mathrm{goo} . \mathrm{gl} / \mathrm{R} 86 \mathrm{bx}>$ y otro más tradicional, una ficha catalográfica: $<$ http://goo.gl/wVPLd $>$.

Inventarios: es el instrumento descriptivo más representativo de un archivo, puede recoger información sobre las series, las unidades de instalación, unidades documentales simples o compuestas, pero de una manera práctica y 
sencilla. Este instrumento tiene como objetivo principal describir con facilidad y de manera ágil la documentación por parte de los archiveros y satisfacer a los usuarios con la información más adecuada de las agrupaciones documentales. Ejemplo de inventario de un archivo municipal $<$ http://goo.gl/3J0S0 $>$.

Estos instrumentos de descripción que se centran en las agrupaciones documentales han sido desde siempre el $a b c$ de los archivos, su soporte era papel en muy diversas dimensiones: libros impresos o manuscritos, fichas, encuadernaciones rudimentarias... pero actualmente no tienen el mismo procedimiento de creación, función, ni tratamiento que hace unos años, puesto que cualquier instrumento generado tiene como punto de partida el tratamiento de la información de una base de datos. Y así, con una descripción adecuada siguiendo la norma ISAD (G) de las distintas agrupaciones se pueden generar a posteriori cualquiera de esos instrumentos, según la demanda, necesidad, $\mathrm{O}$ prioridad del centro ${ }^{19}$.

Pero no sólo se deben de generar recursos denominados genericamente como instrumentos de descripción, también hay que pensar en cómo se van a ofrecer y presentar al personal de los archivos, usuarios y público en general, para ello se dispone de los Sistemas de Acceso respaldados por tecnología web que permiten ofrecer en internet toda esta información albergada y producida en los archivos.

\section{LOS SISTEMAS DE ACCESO, LA WEB Y LAS TECNOLOGÍAS SEMÁNTICAS.}

Si en el siglo pasado unas estanterías o armarios de fichas catalográficas con volúmenes de libros impresos y fichas mecanografiadas destacaban del resto de mobiliario del archivo al ofrecer los instrumentos de descripción de sus agrupaciones documentales. Hoy, se accede a través de un ordenador o dispositivo móvil a esa información recogida en una base de datos, posibilitando su recuperación y uso. Además para el fin que nos ocupa, los archivos, una base de datos contiene las descripciones de las entidades archivísticas y servirá como instrumento de descripción casi único. Las bases de datos se convierten así en un componente fundamental de los denominados Sistemas de Acceso Archivístico o también Sistemas de Información Archivística ${ }^{20}$.

El Sistema de Acceso Archivístico proporciona a los usuarios información sobre las entidades descritas, permitiendo navegar, buscar y recuperar la información deseada, actividades que se realizan a través de medios electrónicos, casi siempre en plataformas web. Lo que afecta a la web, afecta a sus recursos, entre ellos los archivos y sus sistemas de acceso, y por esa razón hay que tener en cuenta factores de usabilidad, accesibilidad, diseño de interfaces de usuario, o reutilización de la información por parte de terceros, por citar algunos elementos. Opciones que se enmarcan en las posibilidades de la web y suponen un nuevo entorno lleno de oportunidades para los archivos y la información descriptiva.

Es indiscutible que la web forma parte de cualquier actividad y ha difundido las descripciones archivísticas como nunca antes había sido posible, pero también han surgido nuevos problemas y hay que plantear cómo se van a ofrecer los recursos y cómo se van a presentar las descripciones en este medio para lograr que cualquier usuario tenga una experiencia satisfactoria. Puesto que la información en la web se puede ofrecer de muchas formas se tiene que encontrar aquella que facilite el uso y la gestión de la información descriptiva, siendo los Sistemas de Acceso Archivísticos las plataformas más apropiadas para ello, puesto que están orientados especialmente a los archivos y no a otros centros o servicios. Los sistemas de acceso son numerosos, al igual que sus posibilidades y funciones, pero comparten y tienen en común las siguientes características básicas:

- Dispone de un interfaz de búsqueda de recursos

- Procesa las consultas de los usuarios

- Recupera las descripciones de las entidades y los recursos digitales disponibles

- Proporciona un interfaz de navegación sobre la información e instrumentos del archivo

- Presta diversos servicios relacionados con el uso, visualización y captura de los datos y documentos ofrecidos

- Cuenta con un apartado de ayuda que posibilita el contacto con los archiveros y otros profesionales

- Diferencia roles de usuarios entre los que destacan los administradores del sistema, profesionales y usuarios ajenos al archivo

Estas características hacen que el Sistema de Acceso Archivístico ofrezca su contenido de manera dinámica, pudiendo modificar la interacción y resultados según las necesidades del usuario o del centro. Todo lo contrario que el soporte papel, donde las descripciones contenidas en las guías o inventarios eran estáticas e inmodificables.

Los Sistemas de Acceso Archivístico se convierten así en una herramienta con múltiples posibilidades de consulta y utilización. Sin olvidar las posibilidades de la web 2.0, donde los usuarios pueden ser partícipes del proceso 
descriptivo añadiendo o corrigiendo información a las agrupaciones documentales u otras entidades archivísticas ofrecidas.

Pero esta información recogida en los archivos y ofrecida por los sistemas de acceso requiere de una estructuración para que pueda ser utilizada de manera estable en la web. Partiendo de ISAD (G) e ISAAR (CPF), como normas internacionales que ofrecen elementos descriptivos, es fácil entender que sus componentes tienen que estar organizados de acuerdo a estándares para que puedan ser usados en redes electrónicas diversas de manera correcta. Para eso surgieron dos normas: EAD Encoded Archival Description (EAD) ${ }^{22}$ y Encoded Archival Context (EAC) ${ }^{23}$, estándares que van a permitir codificar y normalizar en metadatos la estructura de los elementos descriptivos de las entidades. Los instrumentos de descripción tradicionales varían con respecto a autores, elementos utilizados, estilos, o formas de uso, con EAD y otras normas "codificadoras" se estandariza la información del sistema de accesos facilitando su uso en la web gracias a XML.

XML (Extensible Markup Language) ${ }^{24}$ es un lenguaje de marcado que permite estructurar la información descriptiva de un documento textual. Por ejemplo, los elementos descriptivos de una agrupación documental serán los recursos codificados en XML, siguiendo el modelo estructurado estandarizado de EAD. Y como el sistema de acceso ofrece las descripciones codificadas en EAD éstas podrán ser usadas por otras aplicaciones puesto que al codificar cumpliendo sus parámetros se ofrece la información estandarizada a terceros (usuarios o máquinas) bajo las mismas condiciones, relaciones, elementos y estructuras. Aspectos que la norma ISAD (G) o ISAAR (CPF) no desarrollan al centrarse, y ésa es su función, en ofrecer las características, pautas y condiciones para describir las agrupaciones documentales.

Un paso más en la evolución de la tecnología web es hacia una Web Semántica que supone nuevas formas de representar los datos en la red. El funcionamiento de la Web Semántica resulta complejo puesto que requiere el uso de diferentes componentes como bases de datos (único elemento que se dispone hasta ahora), motor de inferencia, ontologías y lenguaje de interrogación respaldados por estándares, protocolos técnicos y lenguajes. Ante tantos se debe señalar que la esencia consiste en acompañar a la información de descripciones explícitas de su significado, descripciones de su estructura interna y de descripciones globales de relaciones y contenido. La clave, una vez más, es la descripción para lograr que toda la información pueda estar enlazada entre sí, favoreciendo un uso universal y sin límites de la misma, logrando un entorno Linked Open Data ${ }^{25}$ o de datos enlazados.

La Web Semántica transforma el concepto tradicional de web, entendida como recursos (páginas web con URL) por un modelo de acceso a datos, independientemente de los sistemas, recursos o direcciones donde se ubiquen, gracias a la coexistencia de información destinada a seres humanos y a maquinas (metadatos / metainformación) en el mismo objeto ${ }^{26}$. Para ello cuenta con RDF (Resource Description Framework) un estándar recomendado por el World Wide Web Consortium de intercambio de datos que favorece el desarrollo de la Web Semántica.

RDF basa su estructura en tres tipos de elementos: recursos (identificados por su individual Uniform Resource Identifier URI), propiedades (atributos), y sentencias (relaciones binarias entre dos elementos). Los cuales descomponen la información en pequeños fragmentos y ofrecen sencillas relaciones semánticas basadas en sentencias del tipo sujeto - predicado - objeto, que componen lo que se denomina tripletas. Para que estas tripletas puedan ser intercambiadas y procesadas por máquinas es necesaria una serialización ${ }^{27}$ de RDF a XML. XML ya se ha nombrado anteriormente con EAC y EAD como lenguaje básico de intercambio y uso de información. Para no alargar más las explicaciones a continuación se muestra de forma mucho más explicita la evolución y posibilidades de las descripciones atendiendo a la tecnología que nos permite la web:

Caso 1) Descripción, mostrada anteriormente, de una agrupación documental.

\begin{tabular}{|l|l|}
\hline Elementos & Información descriptiva \\
\hline 1.1. Código de referencia & ES/AHPZ - A/002446/000004 \\
\hline 1.2. Título & $\begin{array}{l}\text { Expediente del Administrador de las Rentas Estancadas de } \\
\text { Tarazona, Antonio Morales }\end{array}$ \\
\hline 1.3. Fecha(s) & 1886 \\
\hline 1.4. Nivel de descripción & Unidad documental compuesta \\
\hline 1.5 Volumen y soporte de la unidad de descripción & 1 exp. \\
\hline 2.1 Nombre del o de los productor(es) & Administración Provincial de Hacienda de Zaragoza \\
\hline
\end{tabular}

Tabla III. Unidad documental compuesta según ISAD (G). 
Las posibilidades del recurso mostrado en el ejemplo son bastante limitadas, el sistema de acceso lo transmite al usuario y éste lo interpreta para determinar si le interesa o no. Vemos que no hay muchas más funciones puesto que es información textual, carente de una normalización en sus estructuras salvo la determinada por la norma ISAD (G) y mostrada en esa sencilla tabla. No se puede intercambiar y más allá del sistema de archivo que albergue ese contenido, su utilización o aprovechamiento por otras aplicaciones o usuarios puede resultar limitado, y bastante laborioso.

Caso 2) El siguiente ejemplo es un fragmento en XML de la descripción anterior codificada en EAD. Muestra las etiquetas con algunos de sus elementos y atributos ${ }^{28}$.

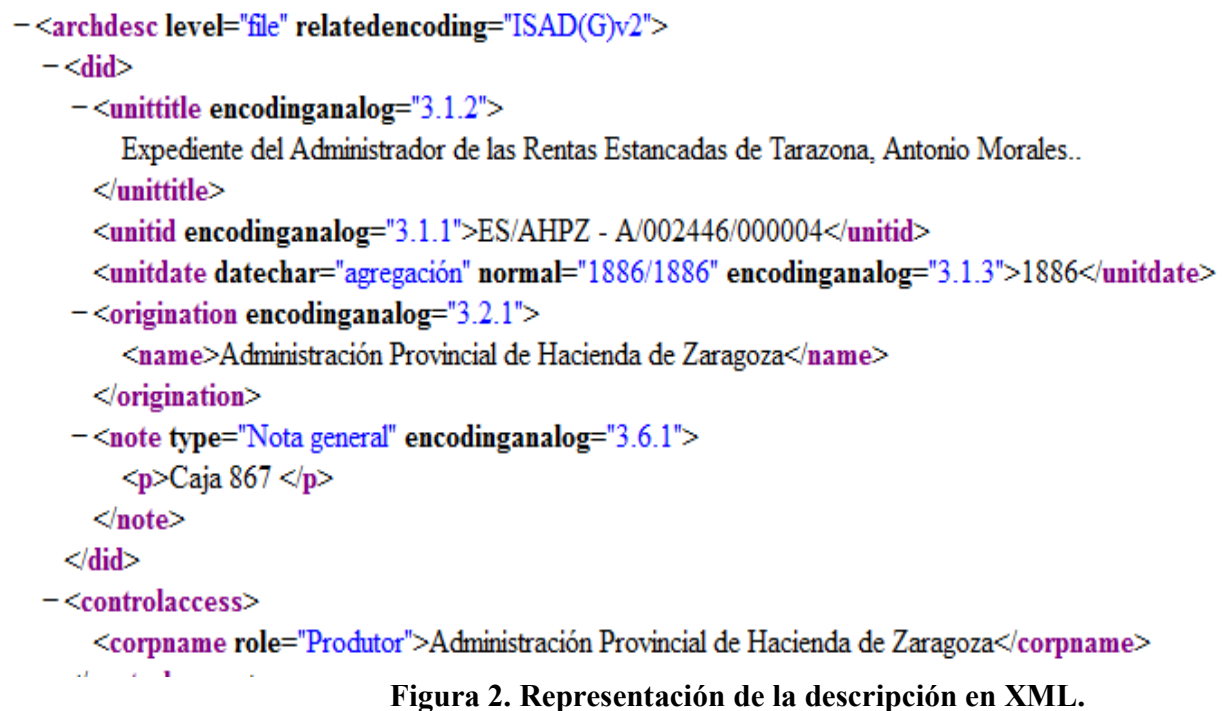

Esta codificación es mucho más rica e interesantes que en el Caso 1. Con el modelo basado en EAD además de poder consultar e interpretar esa información, nos va a permitir la importación y exportación entre sistemas que compartan ese estándar, logrando un posible reaprovechamiento de recursos, interoperabilidad, presentación dinámica, reutilización por otros usuarios y máquinas desde distintos repositorios.

EAD también facilita la presentación de las estructuras siguiendo el modelo multinivel tan importante en los archivos puesto que XML es capaz de establecer relaciones y permitir una navegación entre estructuras anidadas y jerárquicas, representando intelectualmente el contenido para ofrecer una navegación de manera mucho más intuitiva.

Caso 3) Y una variante de esa descripción en una ficticia y sencilla estructura RDF podría ser:

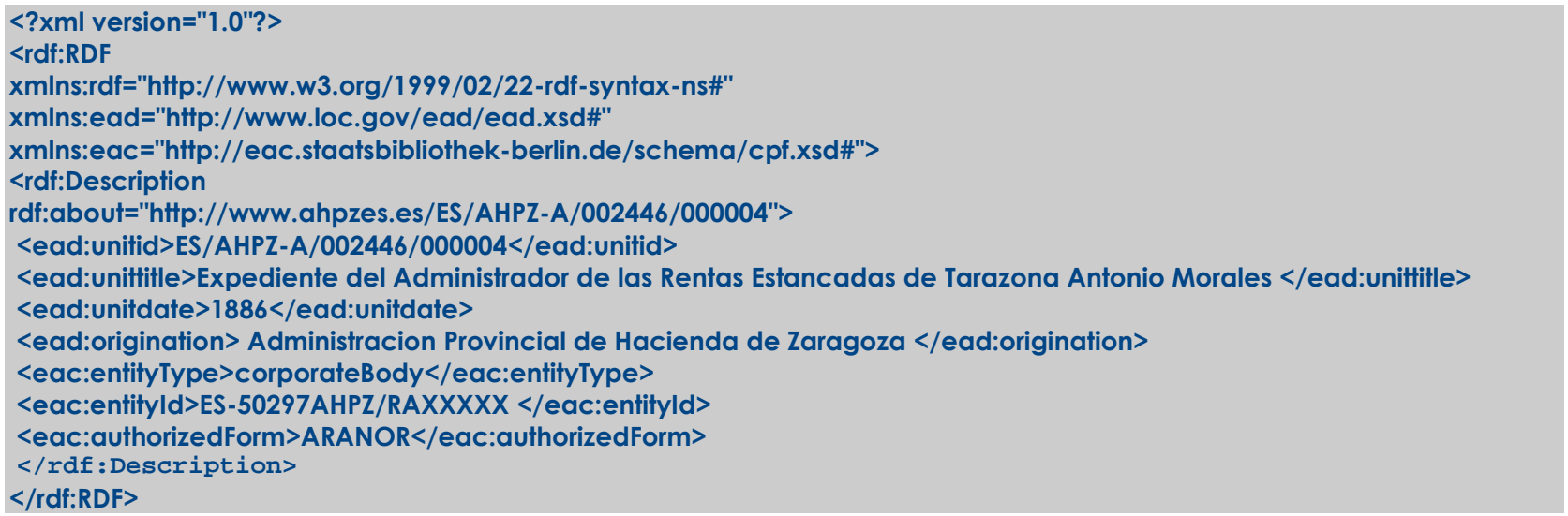

El modelo RDF, que aparentemente no difiere con respecto al ejemplo XML mostrado en el caso 2, aporta mucha más información al desarrollar la estructura en forma de sentencia: sujeto, objeto y predicado. Estas sentencias posibilitan el entendimiento y comprensión por parte de las máquinas de cada elemento de nuestras descripciones. Los elementos descriptivos recogidos en una estructura RDF están identificados, y permiten un intercambio, uso y tratamiento de manera individual. Y si nos fijamos vemos que pueden convivir en un mismo elemento diferentes 
componentes de normas y estándares (ISAD G o ISAAR CPF). Las descripciones recogidas se dividen creando secuencias en RDF que se interpretarán por las máquinas de la siguiente manera:

\begin{tabular}{|c|c|c|}
\hline Sujeto (Recurso) & Objeto (Valor) & Predicado (Propiedad) \\
\hline $\begin{array}{l}\text { http://www.ahpzes.es/ES/AHPZ- } \\
\text { A/002446/000004 }\end{array}$ & http://www.loc.gov/ead/ead.xsd\#unitid & $\begin{array}{c}\text { "ES/AHPZ- } \\
\text { A/002446/000004" }\end{array}$ \\
\hline $\begin{array}{l}\text { http://www.ahpzes.es/ES/AHPZ- } \\
\text { A/002446/000004 }\end{array}$ & http://www.loc.gov/ead/ead.xsd\#unittitle & 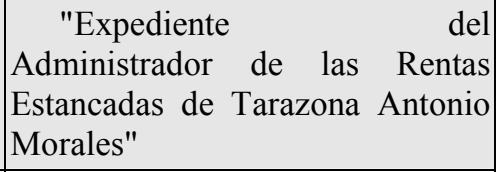 \\
\hline $\begin{array}{l}\text { http://www.ahpzes.es/ES/AHPZ- } \\
\text { A/002446/000004 }\end{array}$ & http://www.loc.gov/ead/ead.xsd\#unitdate & "1886" \\
\hline $\begin{array}{l}\text { http://www.ahpzes.es/ES/AHPZ- } \\
\text { A/002446/000004 }\end{array}$ & ion $h t t p: / / w w w . l o c \cdot g o v / e a d / e a d . x s d \#$ originat & $\begin{array}{l}\text { "Administracion Provincial de } \\
\text { Hacienda de Zaragoza" }\end{array}$ \\
\hline $\begin{array}{l}\text { http://www.ahpzes.es/ES/AHPZ- } \\
\text { A/002446/000004 }\end{array}$ & $\begin{array}{l}\text { http://eac.staatsbibliothek- } \\
\text { berlin.de/schema/cpf.xsd\#entityType }\end{array}$ & "corporateBody" \\
\hline $\begin{array}{l}\text { http://www.ahpzes.es/ES/AHPZ- } \\
\text { A/002446/000004 }\end{array}$ & $\begin{array}{c}\text { http://eac.staatsbibliothek- } \\
\text { berlin.de/schema/cpf.xsd\#entityId }\end{array}$ & $\begin{array}{l}\text { "ES- } \\
\text { 50297AHPZ/RAXXXXX" }\end{array}$ \\
\hline $\begin{array}{l}\text { http://www.ahpzes.es/ES/AHPZ- } \\
\text { A/002446/000004 }\end{array}$ & $\begin{array}{l}\text { http://eac.staatsbibliothek- } \\
\text { berlin.de/schema/cpf.xsd\#authorizedForm }\end{array}$ & "ARANOR" \\
\hline
\end{tabular}

Tabla IV. Representación en RDF.

Y serviría para que estas mismas máquinas puedan "entender" lo siguiente:

\begin{tabular}{|l|l|l|}
\hline Sujeto (Recurso) & Objeto (Valor) & \multicolumn{1}{|c|}{ Predicado (Propiedad) } \\
\hline $\begin{array}{l}\text { El recurso: } \\
\text { ES/AHPZ-A/002446/000004 }\end{array}$ & $\begin{array}{l}\text {..tiene un titulo, según el } \\
\text { elemento "unittitle" del estandar } \\
\text { EAD que es... }\end{array}$ & $\begin{array}{l}\text { las Rentiente del Administrador de Estancadas de Tarazona } \\
\text { Antonio Morales" }\end{array}$ \\
\hline
\end{tabular}

Tabla V. Interpretación fragmento RDF.

La ventaja principal de RDF es la generación de conocimiento e independencia de los elementos que componen la descripción en la web, como hasta ahora no ha sido factible puesto que no lo permite ninguna norma (ISAD G, ISAAR $\mathrm{CPF} . .$.$) ni tampoco ningún estándar (EAC o EAD, por ejemplo).$

Se genera conocimiento puesto que el recurso va a poder ser interpretado por parte de las propias máquinas, identificando su contenido y contexto, pudiendo entender qué es y de qué trata la secuencia de texto en forma de código XML. E independencia, porque el elemento identificado podrá ser utilizado por otros sistemas sin necesidad de recurrir al recurso descriptivo completo, ya que está perfectamente localizado gracias a sus URIs.

\section{CONCLUSIONES}

Gracias a las posibilidades que brinda la tecnología semántica, las descripciones además de poder ser consultadas, interpretadas, o interconectadas, podrán servir para relacionarse con otros elementos y descripciones independientemente del sistema que los albergue, posibilitando el uso y reaprovechamiento automático por otros sistemas. La Web Semántica va a dar un nuevo valor a las descripciones y por primera vez un recurso, además de estar localizado inequívocamente en la web a través de los URI, va a estar definido e identificado, permitiendo a las máquinas saber qué es y cómo ofrecerlo.

La tecnología semántica pretende lograr que las máquinas (conjunto de software y hardware) entiendan la información, mediante la navegación o ejecución de operaciones de forma automática con los recursos de la web, para así poder sacar mayor partido a la información, ahorrando tiempo y esfuerzo a los usuarios al poder ofrecer los resultados que más se aproximen a sus verdaderas necesidades.

Algunos proyectos ya están trabajando con la tecnología semántica desde hace unos años como el proyecto por excelencia en materia cultural de la Unión Europea, Europeana ${ }^{29}$. Y más concretamente en materia de archivos se encuentra Archive $H u b^{30}$ o $E A C-C P F$ Ontology ${ }^{31}$ proyectos que han desarrollado un modelo estructurado de 
información para permitir nuevas formas de uso de las descripciones mediante la aplicación de estándares de datos (y metadatos) basados en tecnología semántica, ofreciendo así la libertad e interacción entre diferentes fuentes de recursos. Como ocurre por ejemplo con el uso de recursos entre Europeana y la recien publicada Digital Public Library of America (DPLA) $)^{32}$.

Estos proyectos dibujan la realidad de la Web Semántica aplicada a los archivos, y muestran que los "datos" cobran más relevancia que los registros descriptivos completos, puesto que un dato puede ser una descripción completa de una entidad archivística, pero también cualquier parte de esa descripción, y ambos pueden ser utilizados sin ningún tipo de distinción.

Si un archivo provee sus descripciones mediante los estándares de la Web Semántica, a través de datos enlazados y correctamente estructurados, además de compartir los recursos va a lograr enriquecer su propia información puesto que sus datos podrían obtener información que mejoren su contenido y contexto, con nuevos aportes provenientes de fuentes diversas: bibliografía desde bibliotecas, contexto histórico desde enciclopedias online, fotografías desde repositorios fotográficos, documentos desde otros archivos, etc. Disponer esta información descriptiva a través de estándares de la Web Semántica va a facilitar la reutilización y aprovechamiento de los mismos por parte de terceros, lo que supondrá dar un nuevo valor a los archivos.

Al leer estas líneas parece que las descripciones archivísticas en un entorno semántico van a necesitar mucha tecnología y elementos que respalden su correcto funcionamiento, pero su base no va a ser puramente tecnológica. El pilar donde se va asentar la Web Semántica está formado por el establecimiento y definición de todas las relaciones entre los diferentes elementos de las entidades descritas, sean Agrupaciones, Agentes, o Funciones, reflejando las normas descriptivas que respaldan los datos, y además identifiquen las entidades, sus asociaciones y cualquier otro componente que represente información archivística. En definitiva, la clave va a estar en estructurar adecuadamente los datos.

Y el paso para estructurar todos estos elementos y determinar sus relaciones ya se ha dado en España con el modelo conceptual fijado por la CNEDA que presenta la información descriptiva siguiendo un esquema entidadrelación, una estructura propia de las bases de datos. Australia o Finlandia también han desarrollado modelos similares con la misma finalidad, poder gestionar la información descriptiva de las entidades archivísticas en conjunto. Pero la importancia de los modelos conceptuales desarrollados en los archivos para la Web Semántica no consiste en presentar los elementos siguiendo el esquema entidad-relación, la importancia de los modelos conceptuales radica en que por primera vez se ofrece la información descriptiva referente a las entidades de los archivos de una manera lógica, estructurada, relacionada y lo que es más importante, interrelacionada. Ahora, para enfocar los modelos conceptuales hacia una Web Semántica se debe de dar un paso más y las relaciones ofrecidas en estos trabajos deben de ser esquematizadas y representadas en los lenguajes apropiados de la web, generando un modelo de datos respaldado por ontologías que hagan posible que cualquier dato descriptivo de cualquier entidad archivística ofrecida por los diferentes sistemas tengan cabida en esta nueva estructura de datos. Si se ha trabajado e invertido enormes esfuerzos y medios en la descripción de miles de recursos, ahora se debe utilizar la tecnología semántica para ayudar a mejorar su difusión y aprovechamiento, dando nuevo valor a las descripciones y por consiguiente a los archivos.

\section{NOTAS}

${ }^{1}$ BONAL ZAZO, J.L.; GENERELO LANASPA, J.J. y TRAVESÍ DE DIEGO, C. Manual de descripción multinivel: Propuesta de adaptación de las normas internacionales de descripción archivística. [En línea]. Disponible en: $<$ http://www.aefp.org.es/NS/Documentos/NormasDescriptivas/MDM2_2006.pdf $>$. [Consulta: 10 de febrero de 2013].

2 SOCIETY OF AMERICAN ARCHIVISTS. Standards for Archival Description: A Handbook. [En línea]. Disponible en: $<$ http://www.archivists.org/catalog/stds99/chapter1.html>. [Consulta: 11 de febrero de 2013].

3 BONAL ZAZO, J.L. La descripción archivistica normalizada: origen, fundamentos, principios y técnicas. Gijón: Trea, 2001, p. 156-159.

${ }^{4}$ BARBADILLO ALONSO, J. Las normas de descripción archivística. Qué son y cómo se aplican. Gijón: Trea. 2011, p. 27.

${ }^{5}$ Ramón Martín Suquía realiza esta definición en un trabajo no publicado sobre descripción archivística.

${ }^{6}$ ISAD (G): Norma Internacional de Descripción Archivística, ISAAR (CPF): Norma Internacional sobre los Registros de Autoridad de Archivos relativos a Instituciones, Personas y Familias, e ISDF Norma internacional para la descripción de funciones.

${ }^{7}$ CONSEJO INTERNACIONAL DE ARCHIVOS. ISAD (G). Norma internacional general de descripción archivística. [En línea]. Disponible en: $<$ http://www.mcu.es/archivos/docs/isad.pdf $>$. [Consulta: 8 de marzo de 2013], p. 16. Aunque el término "descripción archivística" en la versión anglosajona de cada norma es similar (salvo por el uso de un pronombre them en vez de un it en la norma ISDF) en las traducciones españolas de las distintas normas se utilizan expresiones con ligeras diferencias para definir el mismo término.

${ }^{8}$ COMISIÓN DE NORMAS ESPAÑOLAS DE DESCRIPCIÓN ARCHIVÍSTICA. Modelo conceptual de descripción archivística y requisitos de datos básicos de las descripciones de documentos de archivo, agentes $\mathrm{y}$ funciones. [En línea]. Disponible en: 
<http://www.mcu.es/archivos/docs/NEDA MCDA P1 P2 20120618.pdf>. [Consulta: 4 de marzo de 2013], p. 5. Esta Comisión es en la actualidad la máxima autoridad a nivel nacional para desarrollar la estructura y contenido de los datos necesarios para normalizar las descripciones de documentos de archivo, agentes, funciones y archivos. Sus líneas de trabajo se basan, entre otros puntos, en adaptar las normas internacionales (ISAD, ISAAR, ISDF...) a la realidad y necesidades de las instituciones españolas. Para conocer más de esta institución véase $<$ http://www.mcu.es/archivos/MC/CNEDA/Presentacion.html>.

9 Este documento es muy importante para conocer el cambio que se está produciendo en las descripciones de los recursos de archivo. Su importancia radica en que por primera vez se va a normalizar la forma de ofrecer la información de los archivos pasando de la centrada casi exclusivamente en las representaciones de los documentos de archivo, a otra pluridimensional, orientada a la creación y mantenimiento de descripciones de entidades de diferente tipo (documentos de archivo, agentes, funciones, etc.) y de sus interrelaciones. Para conocer más de este documento véase $<$ http://www.mcu.es/archivos/docs/NEDA_MCDA_P1_P2_20120618.pdf>.

${ }^{10}$ Definición basada en CONSEJO INTERNACIONAL DE ARCHIVOS. Op. Cit., p. 17.

${ }^{11}$ Cita de Ramón Martín Suquía extraída de su trabajo no publicado sobre descripción archivística.

${ }^{12}$ Una descripción multinivel se usa para describir un fondo y sus partes. Sin la descripción del primer nivel (fondo), aunque sea sólo con sus elementos mínimos, no se debe presentar la descripción de sus partes (secciones, unidades documentales simples...). BONAL ZAZO, J.L.; GENERELO LANASPA, J.J. y TRAVESÍ DE DIEGO, C. Op. Cit., p. 17.

${ }_{13}$ GUENON, R. El símbolo solsticial de Jano. [En línea]. Disponible en:

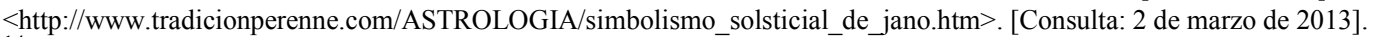

14 Ejemplo similar al utilizado para explicar el IDE del Ayuntamiento de Zaragoza. Ayuntamiento de Zaragoza, Idezar. [En línea]. $<$ http://idezar.unizar.es/ciudad/idezar/informacionIDEs/jerarquia.htm>. [Consulta: 4 de marzo de 2013].

${ }^{15}$ Los ejemplos expuestos están basados en dos ejemplos reales del Archivo Histórico Provincial de Zaragoza pero se han modificado algunos elementos descriptivos para facilitar la explicación.

${ }^{16}$ CELMA, M.; Casamayor, J.C. y Mota, L. Bases de datos relacionales. Madrid: Pearson Educación, 2003.

17 Se vuelve a recordar la definición de descripción: "representar estructuradamente los materiales archivísticos". BONAL ZAZO, J.L.; GENERELO LANASPA, J.L. y TRAVESÍ DE DIEGO, C. Op. Cit., p. 16.

${ }^{18}$ PESCADOR DEL HOYO, M.C. El Archivo: instrumentos de trabajo. Las Rozas, Madrid: Norma, 1993, p. 3.

${ }^{19}$ Véase: CRUZ MUNDEZ, J.R. Manual de Archivística. Madrid: Fundación Germán Sánchez Ruipérez, 2001, p. 256-303.

${ }^{20}$ Sobre sistemas de acceso archivístico véase GARDENER, P.V. System Requirements for Archives Access Systems (v. 1). [En línea]. Disponible en: <http://archivemati.ca/2005/11/04/system-requirements-for-archives-access-systems/>. [Consulta: 18 de marzo de 2013].

Con respecto a los sistemas de información archivística, es una definición utilizada por Ramón Martín Suquía y también se recoge en el texto: SANTAMARIA GALLO, A. La Norma Española de Descripción Archivística (NEDA): análisis y propuestas de desarrollo. [En línea]. Disponible en: $<$ http://www.mcu.es/archivos/docs/NedaAnalisis.pdf>. [Consulta: 13 de febrero de 2013], p. 8.

22 PEIS, E. y RUIZ RODRÍGUEZ, A.A. EAD (Encoded Archival Description): Desarrollo, estructura, uso y aplicaciones. [En línea]. Disponible en: <http://www.hipertext.net>. [Consulta: 18 de marzo de 2013].

${ }^{23}$ ENCODED ARCHIVAL CONTEXT WORKING GROUP. Encoded Archival Context-Corporate Bodies, Persons, and Families (EAC-CPF) Tag Library Appendix ISAAR (CPF) Crosswalk. [En línea]. Disponible en: $<\mathrm{http}: / /$ www3.iath.virginia.edu/eac/cpf/tagLibrary/cpfTagLibrary.html\#d1e8025>. [Consulta: 13 de marzo de 2013].

${ }^{24}$ XML es un metalenguaje muy importante para representar información en la web debido a su gran aceptación, posibilidades y flexibilidad. Véase WORLD Wide Web Consortium. Guía Breve de Tecnologías XML. [En línea]. Disponible en: $<$ http://www.w3c.es/Divulgacion/GuiasBreves/TecnologiasXML >. [Consulta: 19 de marzo de 2013].

${ }^{25}$ Véase: ÁLVAREZ ESPINAR, M. W3C y Linked Open Data. [En línea]. Disponible en: < http://www.w3c.es/Presentaciones/2009/1015interoperabilidadITI-MA/>. [Consulta: 18 de marzo de 2013].

${ }^{26}$ Para conocer más sobre esta tecnología aplicada a la documentación y la gestión de la información se puede consultar: CODINA, Ll. (coord.). Web Semántica y sistemas de información documental. Gijón: Trea, 2009, p. 10. Y más recientemente el boletín especial de ANABAD sobre Web Semántica: NOGUERAS ISO, J., et al. Archivos y gestión de documentos ante la Web Semántica. Boletín de Anabad, 2012, nº 62, p. 21-40.

27 La serialización transforma RDF en formato XML permitiendo la comunicación y el intercambio de información entre diferentes máquinas/aplicaciones gracias a los URIs sin que los datos intercambiados pierdan su sentido, favoreciendo la reutilización, el procesado, la interoperabilidad, y la descentralización de recursos.

${ }^{28}$ Es un ejemplo resumido para conocer más sobre EAD se puede consultar: PEIS, E. y RUIZ RODRÍGUEZ, A.A. EAD (Encoded Archival Description): desarrollo, estructura, uso y aplicaciones. [En línea]. Disponible en: <http://www.hipertext.net>. [Consulta: 12 de marzo de 2013]. También se pueden ver ejemplos en EAD accediendo a cualquier sistema de acceso basado en ICA-atom, por ejemplo <http://demo.icaatom.org/?sf_culture=es>.

${ }_{29}$ Europeana ha construido el modelo de datos Europeana Data Model EDM basado en RDF que permite ofrecer información descriptiva de cualquier recurso, basándose para ello en estándares como OAI-ORE, Dublin Core, SKOS o FOAF entre otros. Refundiendo diversos estándares descriptivos al modelo EDM para favorecer el tratamiento de la información de casi cualquier recurso: libro, imagen, documento o fichero de audio. Véase: EUROPEANA FOUNDATION. Europeana Data Model (EDM) Documentation. [En línea]. Disponible en: <http://pro.europeana.eu/edmdocumentation>. [Consulta: 10 de mayo de 2013].

30 ARCHIVE HUB. Final Product Post: Archives Hub EAD to RDF XSLT. [En línea]. Disponible en: $<$ http://archiveshub.ac.uk/locah/2011/07/01/final-product-post-ead2rdf/>. [Consulta: 11 de mayo de 2013].

31 MAZZINI, S. y RICCI, F. EAC-CPF Vocabulary Specification 1.1. [En línea]. Disponible en: <http://archivi.ibc.regione.emiliaromagna.it/ontology/reference_document/referencedocument.html $>$. [Consulta: 10 de mayo de 2013].

32 PÉREZ, D. Una 'app' española fusiona las dos mayores bases de datos culturales del mundo. [En línea]. Disponible en: <http://goo.gl/AT0kn>. [Consulta: 10 de mayo de 2013]. 


\section{BIBLIOGRAFÍA.}

ARCHIVE HUB. Final Product Post: Archives Hub EAD to RDF XSLT. [En línea]. Disponible en: $<$ http://archiveshub.ac.uk/locah/2011/07/01/final-product-post-ead2rdf/>. [Consulta: 11 de mayo de 2013].

BARBADILLO ALONSO, J. Las normas de descripción archivística. Qué son y cómo se aplican. Gijón: Trea. 2011.

BONAL ZAZO, J.L. La descripción archivistica normalizada: origen, fundamentos, prinicipios y técnicas. Gijón: Trea, 2001.

BONAL ZAZO, J.L.; GENERELO LANASPA, J.J. y TRAVESÍ DE DIEGO, C. Manual de descripción multinivel: Propuesta de adaptación de las normas internacionales de descripción archivística. [En línea]. Disponible en: $<$ http://www.aefp.org.es/NS/Documentos/NormasDescriptivas/MDM2_2006.pdf $>$. [Consulta: 10 de marzo de 2013].

CODINA, L1. (coord.). Web Semántica y sistemas de información documental. Gijon: Trea, 2009.

COMISIÓN DE NORMAS ESPAÑOLAS DE DESCRIPCIÓN ARCHIVÍSTICA. Modelo conceptual de descripción archivística y requisitos de datos básicos de las descripciones de documentos de archivo, agentes y funciones. [En línea]. Disponible en: <http://www.mcu.es/archivos/docs/NEDA_MCDA_P1_P2_20120618.pdf >. [Consulta: 4 de marzo de 2013].

CONSEJO INTERNACIONAL DE ARCHIVOS. ISAD (G). Norma Internacional de Descripción Archivística. [En línea]. Disponible en: <http://www.mcu.es/archivos/docs/isad.pdf $>$. [Consulta: 8 de marzo de 2013].

CONSEJO INTERNACIONAL DE ARCHIVOS. ISAAR (CPF) Norma Internacional sobre los Registros de Autoridad de Archivos relativos a Instituciones, Personas y Familias. [En línea]. Disponible en: $<$ http://www.mcu.es/archivos/docs/isaar.pdf $>$. [Consulta: 8 de marzo de 2013].

CONSEJO INTERNACIONAL DE ARCHIVOS. ISDF Norma internacional para la descripción de funciones. [En línea]. Disponible en: <http://www.mcu.es/archivos/docs/CE/ISDF_ESP_definitiva.pdf $>$. [Consulta: 8 de marzo de 2013].

CONSEJO INTERNACIONAL DE ARCHIVOS. EGAD Resources. [En línea]. Disponible en: $<$ http://www.ica.org/13851/egad-resources/egad-resources.html>. [Consulta: 12 de mayo de 2013].

CRUZ MUNDEZ, J.R. Manual de Archivistica. Madrid: Fundación Germán Sánchez Ruipérez, 2001.

DELGADO GÓMEZ, A. La normalización de la descripción archivística: introducción a Encoded Archival Description (EAD). Cartagena: Archivo Municipal: 3000 Informática, 2003.

MINISTERIO DE EDUCACIÓN, CULTURA Y DEPORTE. Normalización de la descripción archivística: hacia un modelo $\quad$ conceptual. lEn línea]. Disponible en: $<$ http://www.mcu.es/archivos/Novedades/jornada_normalizacion_archivistica.html $>$. [Consulta: 14 de mayo de 2013].

NATIONAL ARCHIVES. The Finnish Conceptual Model for Archival Description (draft) 28.2.2013. [En línea]. Disponible en: <http://goo.gl/ZDFRS>. [Consulta: 14 de mayo de 2013].

NOGUERAS ISO, J.; et al. Archivos y gestión de documentos ante la Web Semántica. Boletín de Anabad, 2012, nº 62 , p. $21-40$.

ENCODED ARCHIVAL CONTEXT WORKING GROUP. Encoded Archival Context-Corporate Bodies, Persons, and Families (EAC-CPF) Tag Library Appendix ISAAR (CPF) Crosswalk. [En línea]. Disponible en: $<$ http://www3.iath.virginia.edu/eac/cpf/tagLibrary/cpfTagLibrary.html\#d1e8025>. [Consulta: 13 de marzo de 2013].

PASTOR SÁNCHEZ, J.A. Tecnologías de la Web Semántica. Barcelona: UOC, 2011.

PESCADOR DEL HOYO, M.C. El Archivo: instrumentos de trabajo. Las Rozas, Madrid: Norma, 1993.

SANTAMARIA GALLO, A. La Norma Española de Descripción Archivística (NEDA): Análisis y propuestas de desarrollo. [En línea]. Disponible en: <http://www.mcu.es/archivos/docs/NedaAnalisis.pdf $>$. [Consulta: 11 de marzo de 2013].

SOCIETY OF AMERICAN ARCHIVISTS. Standards for Archival Description: A Handbook. [En línea]. Disponible en: <http://www.archivists.org/catalog/stds99/chapter1.html>. [Consulta: 5 de marzo de 2013]. 